\title{
Un Strigéide de l'Oie, Apatemon (Australapatemon) anseris n. sp.
}

\author{
Par Georges DUBOIS
}

Parmi les matériels que nous avons reçus de $\mathbf{M}^{11 \mathrm{e}}$ Elisabeth van den Broek, de la * Rijks-Universiteit »d'Utrecht, se trouvait un lot de 47 Strigéides récoltés le 20 mars 1965, à Hollands Diep (l'un des estuaires situés au Sud de Rotterdam), dans le duodénum d'un mâle d'Anser anser (L.). L'Oiseau était bien amaigri, et le contenu de l'intestin localement ensanglanté.

Il nous parut d'emblée que ces Vers n'appartenaient pas à Apatemon gracilis (Rud.), mais qu'ils se rapprochaient beaucoup d' $A$. fuhrmanni Dub., 1937, du Cygne, déjà trouvé aux Pays-Bas par $\mathbf{M}^{\text {He }}$ van den Broek (cf. Dubois 1962, p. 117-118; 1966, p. 32-34) (1). L'espèce dédiée à Fuhrmann est caractérisée par un canal hermaphrodite large et musculeux, plissé tranversalement lorsque le cône génital est rétracté. Mais ce dernier organe, chez le Strigéide de l'Oie, est plus gros en valeurs absolues (580-610/ $370-450 \mu$ au lieu de $310-400 / 210-270 \mu$ ) comme en valeurs relatives, si bien que le rapport des longueurs du segment postérieur et du cône génital est plus faible : 3 à 3,9 (moy. 3,5) au lieu de 3,7 à 5,4 (moy. 4,4) pour fuhrmanni.

Le Strigéide de l'Oie a d'ailleurs été signalé au Kamtchatka, chez Anser fabalis (Lath.) [deux cas d'infestation de l'intestin grêle : 63 et 93 exemplaires], par Ryzhikov, Leonov et Tzimbaluk (1964) qui l'identifièrent à « Australapatemon intermedius (Johnston, 1904) Sudarikov, 1959 ». Le cône génital mesure 440-660/220 $\mu$ (2). Il s'agit sans aucun doute d'Apatemon (Australapatemon) anseris n. sp. Le Ver mesure 3 à $4,49 \mathrm{~mm}$.

(1) Mlle van den Broek nous fit savoir (in litteris, 29 novembre 1965) qu'on trouve peu de Cygnes au Hollands Diep pendant un hiver normal, mais beaucoup d'Oies et des Canards appartenant à diverses espèces. On estime qu'il n'y a que 10 à 20 Cygnus bewicki Yarr., encore moins de Cygnus cygnus (L.) et presque aucun Cygnus olor (Gm.), l'hôte habituel d'Apatemon fuhrmanni.

(2) D'après la figure 3 publiée par les auteurs russes, le cône génital a $300 \mu$ de largeur. Chez Apatemon (Australapatemon) intermedius (Johnston) [d'Australie], l'organe rétracté mesure 640-850/ $420-500 \mu$; le Ver n'a pas des dimensions supérieures à celles du Strigéide de l'Oie (Cf. Dubois et Pearson, 1965). 
Nous remercions vivement $\mathbf{M}^{\text {He }}$ E. van den Broek de nous avoir communiqué quelques-uns des parasites de l'Oie cendrée, sur l'examen desquels la description suivante est basée.

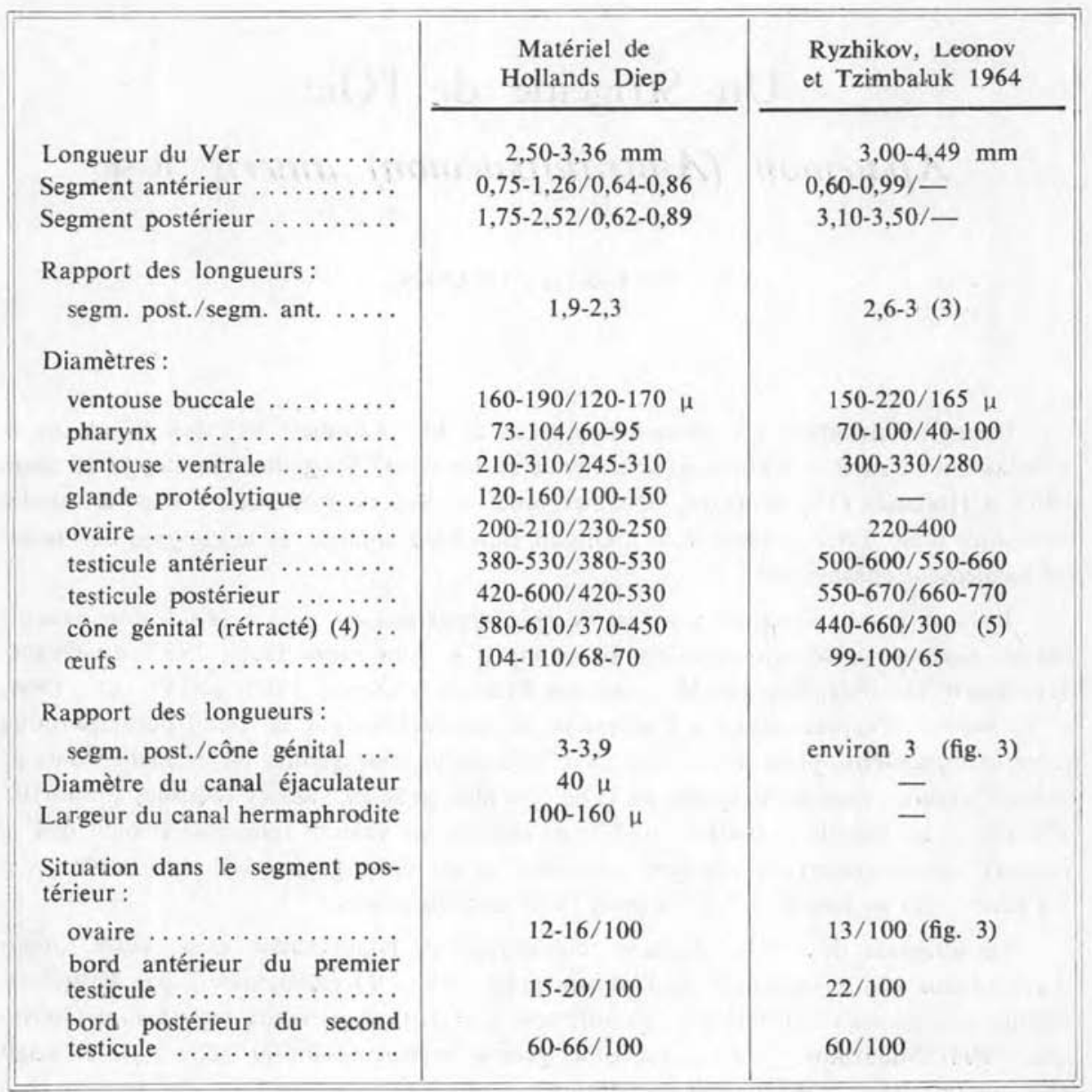

\section{Diagnose}

Segment antérieur utriforme, ovoïde, à ouverture oblique, à bord dorsal curviligne et plus long que le bord ventral souvent rectiligne. Segment postérieur subcylindrique et

(3) D'après la figure 3.

(4) Cône dévaginé : $1.000 / 240-275 \mu$.

(5) D'après la figure 3 , le cône génital mesure $300 \mu$ de largeur (le texte indique $220 \mu$ ). 


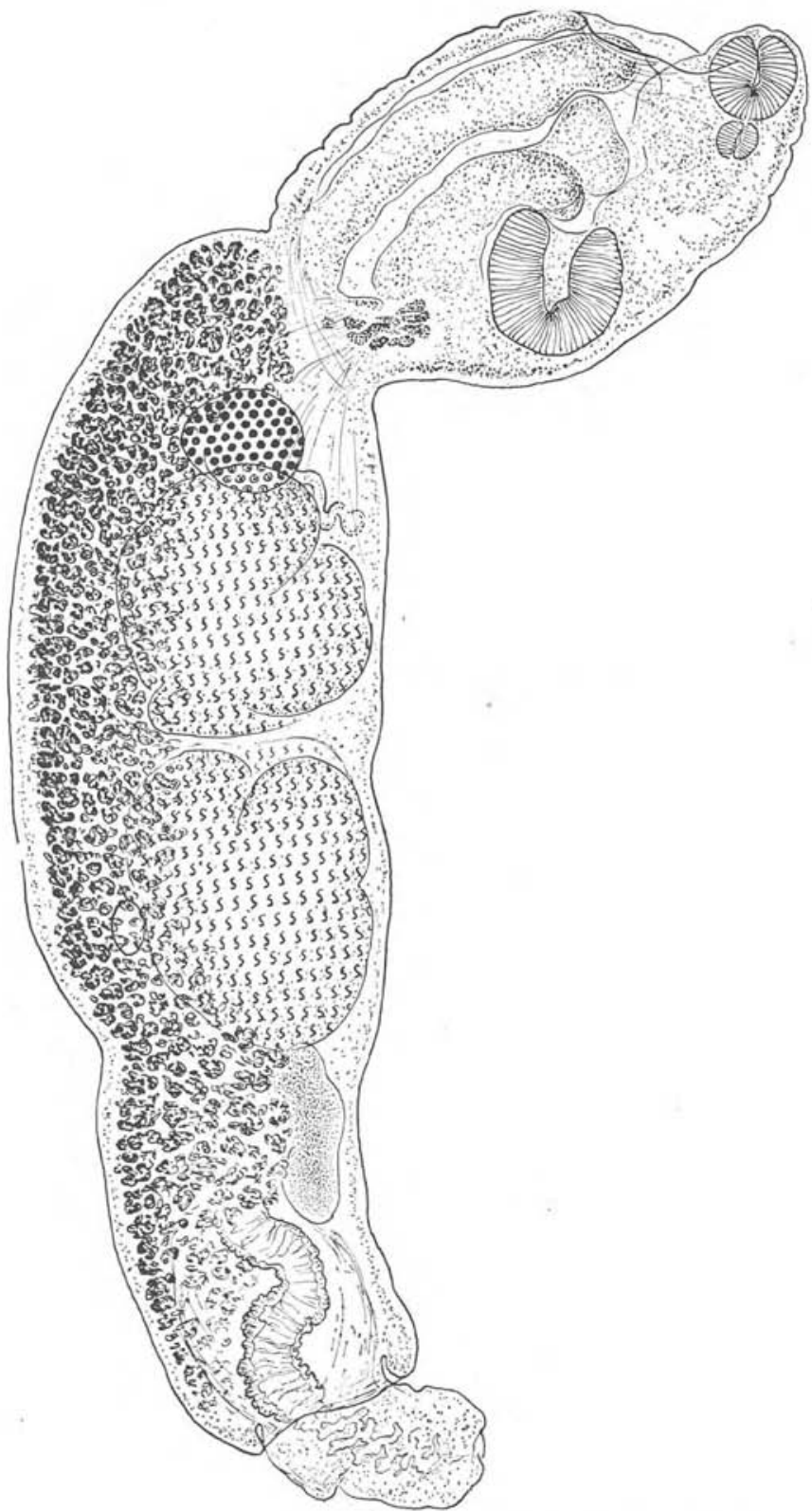

4patemon (Australapatemon) anseris n. sp., de Anser anser (L.). Holotype. Longueur $3,33 \mathrm{~mm}$. Annales de Parasitologie humaine et comparée (Paris), t. 42, 1967, $\mathrm{n}^{\circ} 4$ 
légèrement arqué, formant un angle obtus, parfois droit avec le précédent, dont il est séparé par une constriction bien marquée, - diminuant légèrement de diamètre en arrière des testicules, à extrémité postérieure tronquée perpendiculairement à l'axe longitudinal, où s'ouvre la bourse copulatrice dont la musculature annulaire ( Ringnapf ») est faible. Ventouses bien developpées, la buccale saillante, la ventrale équatoriale et presque deux fois plus grande. Pharynx petit. Glande protéolytique petite, multilobulée, à la base du segment antérieur.

Ovaire réniforme, situé dans le premier cinquième du segment postérieur. Testicules massifs, grossièrement lobés, de forme typique. Vitellogènes s'étendant de la constriction intersegmentaire à l'extrémité du corps, recouvrant obliquement la moitié ventrale du cône génital ovoïde, traversé par un canal hermaphrodite large et sinueux, fortement plissé lorsque le cône est rétracté.

Hôtes : Anser anser (L.) [matériel van den Broek] ; Anser fabalis (Lath.) [Ryzhikov et al.].

Habitat : duodénum et intestin grêle.

Distribution géographique : Europe (Pays-Bas) et Asie (Kamtchatka).

Collections : Institut de Zoologie, Université de Neuchâtel, coll. G. Dubois N ${ }^{\circ} \mathrm{S} 11$ (holotype et paratypes) ; Instituut voor Veterinaire Parasitologie en Parasitaire Ziekten, Rijks-Universiteit, Utrecht, coll. E. van den Broek (paratypes).

\section{Bibliographie}

Dubors (G.), 1938. - Monographie des Strigeida (Trematoda). Mém. Soc. neuchâtel. Sci. nat., VI, 1-535, 354 fig.

-, 1962. - Les Strigeida (Trematoda) de la collection E. van den Broek. Bull. Soc. neuchâtel. Sci. nat., LXXXV, 109-120, 9 fig.

—, 1966. - Du statut de quelques Strigeata La Rue, 1926 (Trematoda). II. Ibid., LXXXIX, 19-56, 6 fig.

- et Pearson (J. C.), 1965. - Quelques Strigeida (Trematoda) d'Australie. Ibid., LXXXVIII, 77-99, 14 fig.

Ryzhikov (K. M.), Leonov (V. A.) et TzimbaluK (A. K.), 1964. - [Nouvel Helminthe d'Oiseaux ansériformes. - Australapatemon skrjabini sp. nov. (Trematoda: Strigeidae)]. Trud. Gelmint. Lab., XIV, 182-186, 3 fig. 